dungen einen ähnlichen Verlauf.“ Einige der großen Praxissoftwarehersteller hätten der KV Telematik GmbH auch bereits signalisiert, dass die D2D-relevanten Anwendungen im ersten Quartal 2016 zur Verfügung stehen werden. Fest steht laut Dreznjak zudem, dass der Terminplan nicht nach hinten verschoben werde.

Aus den Häusern medatixx und CompuGroup ist tatsächlich zu hören, dass die Praxissoftwaresysteme rechtzeitig auf die neuen Vorgaben umgestellt werden. Allerdings stelle das Ende von D2D eine große technische und organisatorische Herausforderung dar, heißt es bei medatixx. Die Umstellung bringe für alle Beteiligten - von den Praxen und KVen über die Datenannahmestellen und Berufsgenossenschaften bis hin $\mathrm{zu}$ den Praxissoftwareherstellern - umfangreiche Änderungen in den täglichen
Abläufen mit sich. Hier hat das Unternehmen den Eindruck, dass die Ärzteschaft bislang kaum über „die von der KV gewünschte Umstellung “ informiert sei. Dennoch begrüßt medatixx, dass die vielfältigen Kommunikationswege der Praxen Schritt für Schritt Richtung KVConnect vereinheitlicht werden sollen. Damit spricht der Softwareanbieter einen wunden Punkt an: Immer wieder wurden in den vergangenen Jahren die fehlende Interoperabilität der Systeme und damit die Schwierigkeiten beim strukturierten Austausch von Daten zwischen den Praxen, kritisiert. Ein Problem, das sogar Einzug ins E-HealthGesetz gefunden hat.

\section{Wer zahlt den Umstieg?}

Bleibt die Frage: Wer finanziert die Umstellung? Zuschüsse von den KVen, de- ren Kommunikationsplattform KVConnect ja eigentlich ist, gibt es nicht. Also wird die Umstellung über die monatlichen Wartungsgebühren der Softwareanbieter oder andere Nutzungsmodelle abgedeckt werden müssen. Bei den medatixx-Systemen wird sich laut dem Unternehmen am bisherigen Prozedere nichts ändern. Dort laufen die KV-Connect-Anwendungen und alle anderen Kommunikationsprozesse der Praxen Schritt für Schritt in die Kommunikationssuite x.comcenter ein, welche die Praxen als kostenpflichtige Zusatzlösung buchen können.

Immerhin wird es bis zum 30. September 2016 einen Parallelbetrieb von D2D und KV-Connect für das elektronische DMP und die übrigen Anwendungen geben.

RebekkaHöhl

\title{
Clevere IT-Lösung für ungeliebte Terminservicestellen?
}

\section{Seit Anfang des Jahres müssen die KVen Terminservicestellen für GKV-Patien- ten einrichten. Hierbei soll sie ein neuer Dienst der KBV unterstützen.}

A nstatt viel Manpower in die Terminvermittlungsstellen zu investieren, könnte die Vergabe von Facharztterminen ganz einfach online laufen. Einen ersten Ansatz dafür bietet der „eTerminservice“, den die KV Telematik GmbH im Auftrag der KBV erarbeitet hat. Technisch sei der eTerminservice so

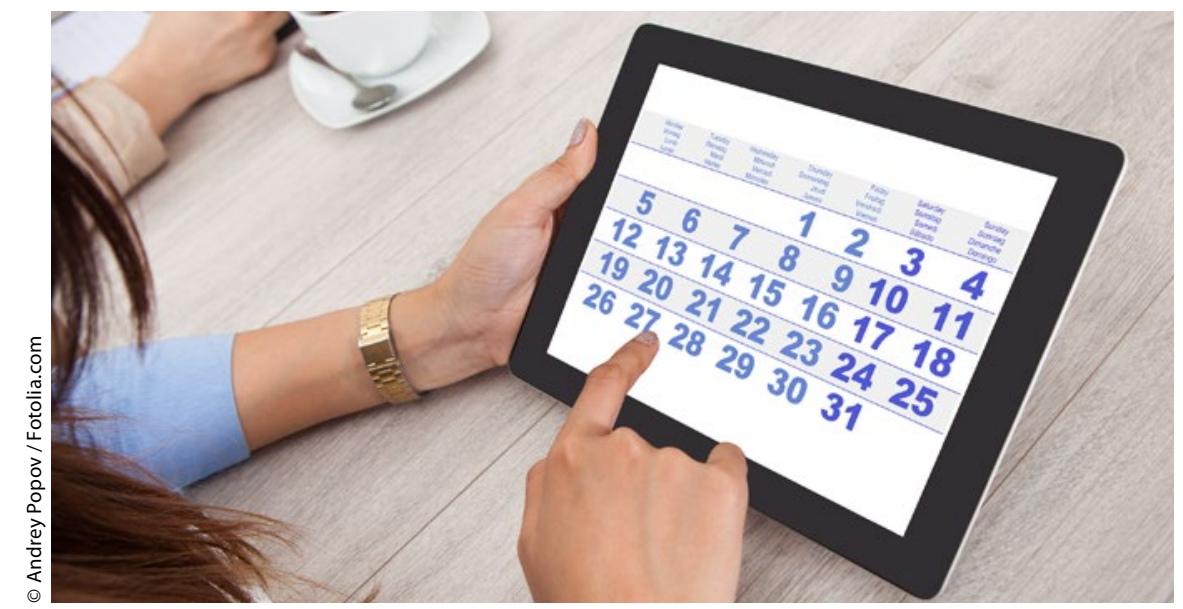

Die Facharztterminvergabe ist auch online möglich.

konzipiert, dass er sowohl in Form einer Web-Anwendung, als auch über eine Schnittstelle direkt aus einem Praxisterminmanagement heraus angesprochen werden könne, erklärt Helena Dreznjak, Leiterin Marketing \& PR bei der KV Telematik GmbH: „Mit letzterer kann die notwendige parallele Pflege mehrerer

URO-NEWS $2016 ; 20$ (2)
Terminkalender vermieden werden." Die technische Spezifikation, die Praxissoftwareanbieter benötigen, um diese Schnittstelle in ihre Systeme zu integrieren, gebe es bereits. Die KV Telematik GmbH will laut Dreznjak aber noch mehr tun: Die Tochtergesellschaft der KBV werde konkret bei den Softwarehäusern nachfragen, ob und wann eine Umsetzung in den Praxissoftwaresystemen geplant ist. Dreznjak: „Unser Wunsch ist, dass dies zum zweiten Quartal hin geschieht, aber es ist seitens der Softwarehäuser eine freiwillige Anwendung." Bei der Web-Anwendung hingegen agieren die KVen und Praxen völlig unabhängig von den Praxissoftwareanbietern. Dafür müssen die Praxen dann aber freie Termine an die KV melden oder in deren System einstellen. Auf diese Lösung will etwa die KV Bremen setzen, indem sie eine gesonderte Homepage für die Terminservicestelle etabliert. Laut Dreznjak wird der eTerminservice von mindestens neun weiteren KVen ebenfalls vollumfänglich durch deren Servicestellen verwendet.

Der eTerminservice soll künftig auch den Patienten als eine Art Online-Terminvergabe zur Verfügung stehen. Den genauen Zeitplan hierfür wird die KV Telematik $\mathrm{GmbH}$ voraussichtlich im $\mathrm{Fe}$ bruar veröffentlichen. Rebekka Höhl 